Mannheimer Manuskripte 145/92

October 1992

\title{
Deformable Media With
}

Microstructure

\section{2}

(T. Ackermann, E. Binz)

Lehrstuhl für Mathematik I

Universität Mannheim

Schloß, D - 6800 Mannheim

W. - Germany

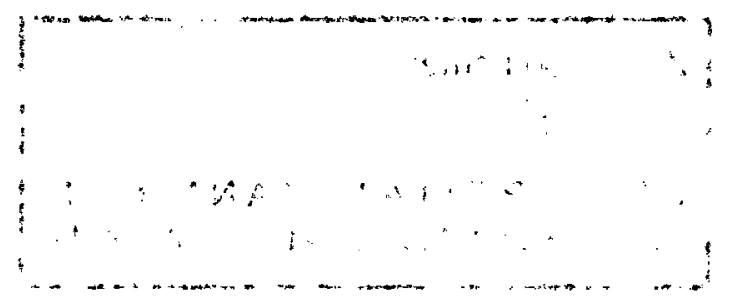




\title{
Deformable Media with Microstructure
}

\author{
T. Ackermann, E. Binz \\ Fakultät für Mathematik und Informatik \\ University of Mannheim
}

\section{Introduction}

In this paper we present a formalism which describes in a global fashion deformations of continua with microstructures. In doing so we have to be aware of the fundamental difference between a local and a global approach.

We present our studies in the following realm of classical physics: We are given a deformable, very thin skin in $\mathbb{R}^{n}$ equipped with a microstructure (called microscopic structure in $[\mathrm{MH}]$ ). Since we deal with skins we assume that the underlying points form a smooth compact manifold, without boundary for simplicity. The microstructure assigns to each point of this manifold well defined microscopic objects such as little rods, directions or vectorspaces. We thus have a fibre bundle over the skin with the objects as fibres. Sections are fields with values in these bundles. Examples in elasticity theory e.g. are various types of Cosserat media. Each of this types of assignments yields to each point a group of symmetries of the respective collection of objects, assumed to be a manifold, a.e. we are given a principal bundle with the symmetry group as structure group. The fibre bundle mentioned is then associated in a natural way.

We emphasize thus merely on the symmetry of the microstructure and not so much on the objects themselves.. This picture is the starting point of our treatment of deformations of this sorts of media.

We equip $\mathbb{R}^{n}$ with a (trivial) principal bundle $P_{\mathbb{R}^{n}}$ of which the structure group $G$ describes the maximal degree of freedom of the microscopic object at each point of the $\mathbb{R}^{n}$. $G$ is assumed to be a Lie group. The microscopic object of the same type sitting on the skin may have less degrees of freedom. Therefore we equip the skin with a principal bundle $P_{\text {skin }}$ with a closed subgroup $H \subset G$ as structure group. Moreover we assume that $P_{\text {skin }}$ is a reduction of the bundle $P_{\mathbb{R}^{n}}$ restricted to the skin.

We now allow the skin to deform. Thus both deform, $P_{\text {skin }}$ and the associated fibre bundle of objects. We handle the deformation of the fibre bundle of objects via the deformation of $P_{\text {skin. }}$. This requires to formulate a mathematical machinery which allows us to describe the deformations of the principal bundle $P_{M}$. In turn this formalism yields a deformation mechanism for the associated bundle (and hence of fields with values in that bundle). This is the content of the first section.

In the second section we recall the description of a medium without microstructure with the help of a first Piola-Kirchhoff stress tensor (cf.[MH], [Bi2]) (without volume correction since this correction is implemented by our integration). We pay special attention to the virtual work caused by a virtual (infinitesimal) deformation and hence on the part of the stress tensor which is irredundant for this work. To what degree the virtual work, however, characterizes the medium itself will not be discussed.

In section three we generalize this apparatus to media with microstructures and point out in section four the influence of the curvature of $P_{s k i n}$ to the physical quality of the medium in a special situation. 
Finally we show that some types of Cosserat media (cf. [MH]) are describable within the frame work presented here, and connect via a (theoretical) example our setting with the theory of liquid crystals. Thereby we will be confronted with the geometry of complex line bundles.

Acknowledgements: We are indebted to Prof. L. Biedenharn for the many discussions on symmetry in physics which are very influential to us. Moreover, we are very thankful to Prof. P. Kramer and Prof. S. Abraham at the University of Tübingen for valuable discussions and hints concerning liquid crystals.

\section{Geometrical background}

The aim of this section is to introduce the geometry underlying our treatment of a deformable medium with microstructure sketched in the introduction. The standard reference for the material presented and the the proofs omitted here is [A]. All maps, bundles, sections and other objects will be taken as smooth, i.e. of class $C^{\infty}$. From the point of view of homotopy theory this gives essentially the same result as the continuous maps would yield and we shall blur the distinction when dealing with homotopy computations. For short let us recall the - more or less - classical approach ( $\mathrm{cf}$. $[\mathrm{MH}]$ ) to media without microstructure: a body $M$ is considered as a finite-dimensional oriented, smooth and compact manifold, without boundary for simplicity, moving and deforming in an ambient euclidean space $\mathbb{R}^{n}$ with a fixed scalar product $\langle$,$\rangle . The configuration space of the theory consists$ of all embeddings $E\left(M, \mathbb{R}^{n}\right):=\left\{j: M \rightarrow \mathbb{R}^{n}\right\}$. Furnished with Whitney's $C^{\infty}$. topology this is a Fréchet-manifold (cf. [BSF]). In the following let $m(j)$ and $\mu(j)$ denote respectively the Riemannian metric on $M$ given as the pullback of $\langle$,$\rangle on$ $\mathbb{R}^{n}$ by $j \in E\left(M, \mathbb{R}^{n}\right)$ and the induced volume form on $M$.

To describe a deformable medium with microstructure we assign - in analogy to gauge theories of elementary particles - 'internal degrees of freedom' to each point of the embedded body yielding $P_{M}$ and the ambient space yielding $P_{\mathbb{R}^{n}}$ as well. We assume thereby that these degrees of freedom do not vary from point to point: i.e. we are given a Lie group $H$ reflecting the internal properties of the body (e.g. $H=\operatorname{SO}(n)$ cf. sec.5) and a Lie group $G$ for the ambient $\mathbb{R}^{n}$ reflecting the external degrees of freedom. While $P_{\mathbb{R}^{n}}:=\mathbb{R}^{n} \times G$ is a trivial principal bundle over $\mathbb{R}^{n}$ we require $P_{M}$ to be a not necessary trivial $H$-principal bundle. We want to generalize this situation slightly: we replace the ambient $\mathbb{R}^{n}$, by a finite-dimensional Riemannian manifold $N$ and the 'enlarged ambient space' $\mathbb{R}^{n} \times G$ by a $G$-principal bundle $P_{N}$ over $N$. Let $\pi^{M}$ and $\pi^{N}$ denote the respective projection from $P_{M}$ to $M$ and from $P_{N}$ to $N$. Therefore we end up with the following 'dictionary' for a deformable medium:

\begin{tabular}{l|r|r}
. & without microstructure & with microstructure \\
\hline body & embedded manifold $M$ & $H$-principal bundle $P_{M} \stackrel{\pi_{M}}{\longrightarrow} M$ \\
ambient space & Riemannian manifold $N$ & $G$-principal bundle $P_{N} \stackrel{\pi_{N}}{\longrightarrow} N$ \\
configuration & embedding $j: M \rightarrow N$ & principal embedding $J: P_{M} \rightarrow P_{N}$
\end{tabular}

To understand this we recall that a principal embedding $J: P_{M} \rightarrow P_{N}$ means a fibre preserving embedding $P_{M} \smile P_{V}$ which is a bundle morphism and respects 
the group-actions with respect to a given monomorphism $H \hookrightarrow G$ of the Lie groups
(cf.[KN]).

A principal embedding $J: P_{M} \rightarrow P_{N}$ induces an embedding $j: M \rightarrow N$ between the corresponding base manifolds. On the other hand, given an embedding $j: M \rightarrow N$ of the bases, it isn't obvious at all that there exists a principal embedding $J$ which induces $j$. At least in the compact case - a.e. if $M$ is a compact manifold without boundary, and $H, G$ are compact Lie groups - we get a sufficient criterion: certain obstructions $\alpha^{i} \in H^{i}\left(M, \pi_{i-1}(G / H)\right)$ have to vanish (cf. [A]).

In the following let $M, N$ be compact manifolds without boundary such that $\operatorname{dim} N \geq \operatorname{dim} M$. For the application below we set $N=\mathbb{R}^{n}$. The Lie groups $G$ and $H$ with $H \subset G$ being closed are assumed to be compact. Furthermore we suppose to have a principal embedding $J \in E_{j}\left(P_{M}, P_{N}\right)$ of the corresponding principal bundles, which induces an embedding $j \in E(M, N)$ on the base manifold $M$. Obviously it is true that the pullback-bundle $j^{*} P_{N}:=\left\{(m, p) \in M \times P_{N} \mid j(m)=\pi_{N}(p)\right\}$ is again a $G$-principal bundle. By definition (cf. $[\mathrm{KN}]$ ) a principal embedding of $P_{M}$ into $P_{M}$ over the identity is called a reduction. We have the following at hand (cf. $[\mathrm{A}]$ ):

\section{Lemma 1.1}

Let $P_{M}$ and $P_{N}$ be given principal bundles over $M$ and $N$, respectively. $R\left(j^{*} P_{N}, P_{M}\right)$ denotes the collection of all reductions of the $G$-principal bundle $j^{*} P_{N}$ to the $H$-principal bundle $P_{M}$. The map $I_{j}: R\left(j^{*} P_{N}, P_{M}\right) \rightarrow E_{j}\left(P_{M}, P_{N}\right)$ defined by $\mathrm{I}_{j}(r)=\iota_{j} \circ r$ for all $r \in R\left(j^{*} P_{N}, P_{M}\right)$ is a bijection. The map $\iota_{j}: j^{*} P_{N} \rightarrow P_{N}$ is the canonical map defined by the pullback.

The advantage of the previous Lemma is evident: the information about the principal embeddings $E\left(P_{M}, P_{N}\right)$ is 'encoded' entierly in the reductions $R\left(j^{*} P_{N}, P_{M}\right)$ with $j \in E(M, N)$ ! We shall start to analyse reductions more closely (cf. [KN]):

To this end let $Q \stackrel{\pi}{\longrightarrow} M$ any $G$-principal bundle and $H \hookrightarrow G$ a closed subgroup. It is a standard fact that there is a bijective correspondence between $H$-principal bundles $P$ which are reductions of the $G$-principal bundle $Q$ and sections of the associated bundle $E:=Q \times_{G} G / H$ with homogeneous fibre $E_{x}=G / H$. In particular, $Q$ is reducible to a $H$-principal bundle iff $E$ admits a section. The idea is as follows: Because there is an isomorphism $E \cong Q / H$ the bundle morphism $\mu: Q \rightarrow Q / H$ over $i d_{M}$ defines a $H$-principal bundle. Given a section $\sigma \in \Gamma^{\infty}(E)$ the pullback-bundle $\sigma^{*} Q$ is a reduced bundle of $Q$.

Let $\Sigma_{\sigma}:=\left\{\tau \in \Gamma^{\infty}(E) \mid \tau^{*} Q \cong \sigma^{*} Q\right\} \subset \Gamma^{\infty}(E)$ be the set of those sections of $E$ which induce isomorphic $H$-principal bundles over $M$.

\section{Remark}

Homotopic sections $\tau_{1}, \tau_{2} \in \Gamma^{\infty}(E)$ induce isomorphic bundles $\tau_{1}^{*} Q \cong \tau_{2}^{*} Q$. Here 'homotopic' is understood as 'fibre-homotopic', i.e. the homotopy $h: M \times[0,1] \rightarrow E$ is a section for every $t \in[0,1]$. It isn't true that an isomorphism $\alpha^{*} Q \cong \beta^{*} Q$ yields
a homotopy $\alpha \simeq \beta$.

It is not hard to check that $\Gamma^{\infty}(E)$ equipped with the $C^{\infty}$-topology is a differentiable Fréchet-manifold which in general is not connected: the connected component of $\gamma \in \Gamma^{\infty}(E)$ is determined by the homotopy classes of sections $U_{\gamma}:=\left\{\tau \in \Gamma^{\infty}(E) \mid \tau \simeq \gamma\right\}$. By definition these are open in $\Gamma^{\infty}(E)$ and we have 
$\Sigma_{\sigma}=\bigcup_{\gamma} U_{\gamma}$, where $\gamma$ runs through $\left\{\tau \in \Gamma^{\infty}(E) \mid \tau^{*} Q \cong \sigma^{*} Q\right\}$. Along the lines the following is proved in $[\mathrm{A}]$ :

\section{Theorem 1.2}

The subset $\Sigma_{\sigma}$ of $\Gamma^{\infty}(E)$ together with the induced topology is a smooth Fréchetmanifold.

We have not mentioned yet one further structure which will be important for our purpose, cf. sec.3: the group of gauge transformations. Given a $G$-principal bundle $Q$ we recall that the group of gauge transformations $\mathcal{G}_{Q}$ might be equivalently understood as the group of (principal) bundle automorphisms of $Q$ covering the identity on the base manifold $M$ or as sections of the associated group-bundle $P \times_{G} G$, here $G$ acts on itself by conjugation (cf. e.g [BSF]). Endowed with Whitney's $C^{\infty}$ topology this is a (infinit dimensional) smooth Fréchet-Lie group with Lie algebra $\Gamma^{\infty}\left(P \times_{A d} g\right)$ - where $g$ denotes the Lie algebra of $G$ and $A d: G \rightarrow \operatorname{Aut}(g)$ is the adjoint representation. The group $\mathcal{G}_{Q}$ acts transitively and Fréchet-differentiable from the left on $\Sigma_{\sigma}$ (cf. [A]). This is a consequence of the so called $\Omega$-lemma (cf.[P]). Consequently $\Sigma_{\sigma}$ is a homogeneous Fréchet-manifold. However this action is not free. Let $I_{\sigma}$ be the isotropy group, a smooth Fréchet Lie group. In summarizing we state

\section{Theorem 1.3}

a) The Fréchet-Lie group $\mathcal{H}_{\sigma^{*} Q}$ - the group of gauge transformations of $\sigma^{*} Q-$ is Fréchet-diffeomorphic to the isotropy group $I_{\sigma}$ and therefore a differentiable Fréchet-Lie subgroup of $\mathcal{G}_{Q}$.

b). The quatient $\mathcal{G}_{Q} / \mathcal{H}_{\sigma * Q}$ endowed with the induced structure is a differentiable Fréchet-manifold which is Fréchet-diffeomorphic to $\Sigma_{\sigma}$.

c) The quotient map $\mathcal{G}_{Q} \rightarrow \mathcal{G}_{Q} / \mathcal{H}_{\sigma^{*} Q}$ defines a differentiable $\mathcal{H}_{\sigma^{*} Q^{-F r e ́ c h e t-~}}$ principal bundle.

We now return to the study of the manifold of principal embeddings $E\left(P_{M}, P_{N}\right)$. There is a natural map $\Pi: E\left(P_{M}, P_{N}\right) \rightarrow E(M, N)$ which is in general not surjective, cf. the above remark, and therefore in general we can't write $E\left(P_{M}, P_{N}\right)=\bigcup_{j \in E(M, N)} E_{j}\left(P_{M}, P_{N}\right)$. Let $\mathcal{E}_{P_{M}}:=\left\{j \in E(M, N) \mid j^{*} P_{N} \cong\right.$ $\left.P_{M} \times{ }_{H} G\right\}$ consists of embeddings such that the pullback of $P_{N}$ is isomorphic to the $G$-principal bundle $P_{M} \times_{H} G$. It is well known that those $G$-principal bundles $j^{*} P_{N}$ with $j \in \mathcal{E}_{P_{M}}$ can be reduced to $P_{M}$ (cf. [KN]). Reasoning analogously as in the proof of the above Theorem 1.2 we get:

\section{Theorem 1.4}

The subset $\mathcal{E}_{P_{M}} \subset E(M, N)$ together with the induced structure is a differentiable Fréchet-manifold.

Now obviously it is true that $E\left(P_{M}, P_{N}\right)=\bigcup_{j \in \mathcal{E}_{P_{M}}} E_{j}\left(P_{M}, P_{N}\right)$ and that the induced map $\bar{\Pi}: E\left(P_{M}, P_{N}\right) \rightarrow \mathcal{E}_{P_{M}}$ is surjective. We conclude

\section{Theorem 1.5}

The map $\bar{\Pi}: E\left(P_{M}, P_{N}\right) \rightarrow \mathcal{E}_{P_{M}}$ defines a differentiable Fréchet-fibre bundle whose fibres $E_{j}\left(P_{M}, P_{N}\right)$, depending on the connected components of $\mathcal{E}_{P_{M}}$, are equal

$$
R\left(j^{*} P_{X} . P_{.1}\right)=\mathcal{G}_{j^{*} P_{N}} / \mathcal{H}_{P_{M}} .
$$


The idea of the proof is the following (cf. [A]): The local triviality is an easy consequence of the local convexity of the Fréchet manifold $\mathcal{E}_{P}$. To show the second part $E_{j}\left(P_{M}, P_{N}\right)=\mathcal{G}_{j^{*} P_{M}} / \mathcal{H}_{P_{M}}$ we use Lemma 1.1 and (b) in Theorem 1.3.

\section{Remark}

There is no essential difference working with Hilbert- resp. Banach-manifolds instead of Fréchet-manifolds (cf. [A]) in the above context.

One important example of this kind of structures concerns principal embeddings of orthonormal frame bundles. At least in this case $\mathcal{E}_{P_{M}}=E(M, N)$ and $\bar{\Pi}=\Pi$ : $E\left(P_{M}, P_{N}\right) \rightarrow E(M, N)$ defines a differentiable Fréchet bundle (cf. [A]).

Another example are principal embeddings of trivial bundles. Here for every $j \in$ $E(M, N)$ there can be constructed fibre wise a $J(j)$ with the help of the inclusion $i: H \hookrightarrow G$. Moreover the action of the group of gauge transformations yields all of $E_{j}\left(P_{M}, P_{N}\right)$, cf. Theorem 1.3. Consequently in this case $\bar{\Pi}=\Pi: E\left(P_{M}, P_{N}\right) \longrightarrow$ $E(M, N)$ defines a differentiable Fréchet bundle, too.

Let us make some remarks concerning induced embeddings of associated vectorbundles. More precisely, let $F$ be a finite-dimensional for simplicity compact manifold and $E \subset F$ a submanifold. Let us assume furthermore that $G$ operates on $F$ and that the operation of $H \subset G$ restricts to an operation of $H$ on $E \subset F$. Clearly this assumption is void if $H=G$. In particular we have specified homomorphisms $\varphi_{H}: H \rightarrow$ Diff $E$ and $\varphi_{G}: G \rightarrow$ Diff $F$. We construct the associated bundles $\mathbb{E}:=P_{M} \times_{H} E$ over $M$ and $\mathbb{F}:=P_{N} \times_{G} F$ over $N$. Every principal embedding $J \in E\left(P_{M}, P_{N}\right)$ together with the inclusion map $i: E \hookrightarrow F$ extends obviously to an embedding of the trivial bundles $P_{M} \times E$ into $P_{N} \times F$. This extension induces embeddings $\bar{J}$ of the associated bundles $\mathbb{E}$ into $\boldsymbol{F}$, which are well defined due to the $H$-equivariance of $J$. In summarizing we state:

\section{Lemma 1.6}

Under the previous assumptions every $J \in E\left(P_{M}, P_{N}\right)$ induces an embedding $\bar{J} \in E_{H}(\mathbb{E}, \mathbb{F})$ of the associated bundles $\mathbb{E}$ and $\mathbb{F}$.

Let $L(\mathbb{E}, \mathbb{F}):=\{F \mid F: \mathbb{E} \rightarrow \mathbb{F}\}$ denote the bundle morphisms of $\mathbb{E}$ to $\mathbb{F}$. As a consequence of the previous lemma we obtain

\section{Corollary 1.7}

The map $E\left(P_{M}, P_{N}\right) \stackrel{\mathcal{J}}{\longrightarrow} L(\mathbb{E}, \mathbb{F})$ defined by $J \mapsto \bar{J}$ is injective if $\varphi_{G}$ is injective, more precisely $\mathcal{J}: E\left(P_{M}, P_{N}\right) \rightarrow \mathcal{J}\left(E\left(P_{M}, P_{N}\right)\right) \subset L(\mathbb{E}, \mathbb{F})$ is a smooth diffeomorphism if $G$ operates without fixpoints on $E$. The canonical projection from $\mathcal{J}\left(E\left(P_{M}, P_{N}\right)\right)$ to $\mathcal{E}_{P_{M}}$ is surjective.

\section{Remark}

Let us replace the symbol $\mathcal{J}\left(E\left(P_{M}, P_{N}\right)\right)$ by $\operatorname{Ind}(\mathbb{E}, \mathbb{F})$. The latter space consists of all embeddings of $\mathbb{E}$ into $\mathbb{F}$ induced by the maps in $E\left(P_{M}, P_{N}\right)$. 


\section{The characterization of a deformable medium without microstructures}

The medium, a skin, deformable in the manifold $N$ with Riemannian metric $\langle$, is assumed to have the shape of a manifold; in fact this manifold is assumed to be the image of a smooth embedding $j$ of an abstract manifold $M$.

We recall that $M$ is supposed to be a smooth, compact, oriented and connected manifold and for simplicity without boundary. The embedding $j$ is called a configuration, the space of all configurations is denoted by $E(M, N)$ and is assumed to be endowed with the $C^{\infty}$-topology. That is to say $E(M, N)$ is a Fréchet manifold. The quality of a medium without microstructure is characterized by a smooth $T N$ valued one-form $\alpha(j)$, depending on $j$. This one-form is called the first PiolaKirchhoff stress tensor or simply a stress form of the medium. The virtual work $\mathcal{F}(j)(l)$ at the configuration $j$ caused by any pointwise performed (infinitesimal) distortion $l \in C_{j}^{\infty}(M, T N)$ is described by

$$
\mathcal{F}(j)(l)=\int_{M} \alpha(j) \bullet \nabla l \mu(j) \quad \forall j \in E(M, N), \forall l,
$$

with $\nabla$ denoting the covariant derivative of $\langle$,$\rangle on N$. Here $C_{j}^{\infty}(M, T N)$ denotes the collection of all the smooth maps from $M$ into $T N$ for which $\pi_{N} l=j$, that is $\left.C_{j}^{\infty} / M, T N\right)=T_{j} E(M, N)$ for any $j \in E(M, N)$. For the dot product involved in the integrand we refer to the appendix. A reformulation of this virtual work reads as

$$
\mathcal{F}(j)(l)=\int_{M}\left\langle\nabla^{*}(j) \alpha(j), l\right\rangle \mu(j)
$$

* with $\nabla^{*}\left(j^{\prime}\right)$ the divergence operator determined by the Levi-Cività connection $\nabla(j)$ of the metric $m(j)$ (cf. appendix). $\nabla^{*} \alpha(j)$ is called the (internal) force density $\Phi(j)$ at $j$. Solving

$$
\Phi(j)=\nabla^{*} \alpha(j)=\Delta(j) h(j)
$$

with $\Delta(j)$ being the Laplacian determined by $m(j)$ we find a smooth map

$$
h(j) \in C_{j}^{\infty}(M, T N)
$$

depending on $j$ (cf. [Bi2]). Therefore the virtual work rewrites as

$$
\mathcal{F}(j)(l)=\int_{M} \nabla h(j) \bullet d l \mu(j) \quad \forall j \in E(M, N), \forall l \in C_{j}^{\infty}(M, T N) .
$$

For each $j \in E\left(M, \mathbb{R}^{n}\right)$ the covariant derivative $\nabla h(j)$ of $h(j)$, called the pseudo exact part of $\alpha(j)$, is the part irredundant with respect to the virtual work $\mathcal{F}$. This yields for each $j$ a decomposition $\alpha(j)=\nabla h(j)+\beta(j)$ with $\beta(j):=\alpha(j)-\nabla h(j)$, orthogonal (with respect to the dot metric (cf. appendix)) to $\nabla h(j)$. For all this we refer to $[\mathrm{Bi} 2]$ and $[\mathrm{Bi} 3]$.

In what follows we characterize the global properties of the medium only up to its influence to the virtual work; i.e. we take $\mathcal{F}$ as the characteristic ingredient. 


\section{A general frame-work for deformable media with or without microstructure}

In this section we develop the formalism with which we treat media with or without microstructures. To this end let $F$ and $E \subset F$ be compact, smooth, oriented and connected manifolds. $E$ and $F$ are the collections of microscopic objects on $\mathbb{R}^{n}$ and on $M$, respectively. To generalize the virtual work introduced in the section above, we introduce a metric $\langle,\rangle_{F}$ on $F$. The manifold $\mathbb{R}^{n} \times F$ carries the product metric, a.e. a metric (, ) on all of $\mathbb{R}^{n} \times F$ given as follows: let $p r_{\mathbb{R}^{n}}: \mathbb{R}^{n} \times F \rightarrow \mathbb{R}^{n}$ and $p r_{F}: \mathbb{R}^{n} \times F \rightarrow F$ denote the projections onto the first and the second factor, respectively. For any $v, w \in T_{(z, f)}\left(\mathbb{R}^{n} \times F\right)$ we set

$$
(v, w):=\left\langle T p r_{\mathbb{R}^{n}} v, T p r_{\mathbb{R}^{n}} w\right\rangle+\left\langle T p r_{F} v, T p r_{F} w\right\rangle_{r} .
$$

Moreover, let $G$ and $H \subset G$ be the respective isometry groups of $F$ and $E$. Their respective Lie algebras are $\mathbf{g}$ and $\mathbf{h}$. If the microscopic objects on $M$ and $\mathbb{R}^{n}$ would form a vector space, say $E^{\prime}$ and $F^{\prime}$, respectively, with $E^{\prime} \subset F^{\prime}$, then $E$ and $F$ could be unit spheres with respect to a fixed scalar product in $F^{\prime}$.

The group $G$ reflects the maximal degree of freedom of the given type of microscopic objects on $\mathbb{R}^{n}$, while as $H$ on the other hand describes the degree of freedom of the microscopic object on $M$. If $\operatorname{dim} F=0$ then we have no microstructure at hand and we are back in the realm of sec.2.

If we think of the body to have attached at each point the manifold $E$ of microscopic object we have a fibre bundle over the body. If this body deforms, then the whole fibre bundle deforms. This deformation of the fibre bundle can be handled by describing the deformation of a principal bundle with $H$ as structure group and in turn by describing the deformation of the fibre bundle via the process of association. This is the main reason why we put the concept of symmetry into the foreground that much and why we have developed the geometric apparatus in sec. 1 on such an abstract level.

First, we describe the deformation of principal bundles, that is we describe the space of configurations of symmetry in a simple situation and relate them to embeddings of the associated bundle: With the groups $H$ and $G$ we form the trivial bundles $M \times H$ and $\mathbb{R}^{n} \times G$. Any smooth map $s: M \longrightarrow G$ can be referred to as a configuration of symmetry. We relate this sort of configuration with the geometric configuration $j \in E\left(M, \mathbb{R}^{n}\right)$ via the smooth principal embedding

$$
J(j): M \times H \longrightarrow \mathbb{R}^{n} \times G
$$

given by

$$
J(j)(q, h)=(j(q), s(q) h)
$$

for any $q \in M$ and any $h \in H$. Thus $J(j)$ subsumes both the configuration of symmetry and the geometric configuration $j$. We refer therefore to $J(j)$ as a configuration of symmetry of our medium with microstructures. Vice versa any equivariant smooth embedding $J(j): M \times H \rightarrow \mathbb{R}^{n} \times G$ over some $j \in E\left(M, \mathbb{R}^{n}\right)$ can be obtained via a smooth map $s: M \longrightarrow H$ given by $s(q)=J(j)^{-1}(j(q), J(j) \mathbf{1})$ where $\mathbf{1} \in G$ denotes the unit element and $q$ varies in $M$. Each $J(j)$ defines

$$
\bar{J}(j): M \times E \rightarrow \mathbb{R}^{n} \times F,
$$


a smooth embedding of the associated bundles by setting

$$
\bar{J}(j)(q, e)=(q, s(q) e)
$$

for all $q \in M$ and all $e \in E$.

Since in general principal bundles over $M$ are not trivial, we extend our notion of a configuration of symmetry of a medium with microstructures as follows: Let $P_{M}$ be an oriented principal bundle with $H$ as its structure group and $\pi^{M}: P_{M} \rightarrow M$ as its projection. By a configuration of symmetry we mean a smooth principal embedding

$$
J: P_{M} \longrightarrow \mathbb{R}^{n} \times G .
$$

Clearly $j\left(\pi^{M}(p)\right)=p \mathbb{R}_{\mathbb{R}^{n}} \circ J(p)$ for all $p \in P_{M}$ defines $j \in E\left(M, \mathbb{R}^{n}\right)$. This is equivalent to say that a configuration of symmetry in this general setting is a reduction of $j^{*}(M \times G)$ (cf. sec.1). As shown in sec.1 the space of all configurations of symmetry of our deformable medium with microstructures is the Fréchet manifold $E\left(P_{M}, \mathbb{R}^{n} \times G\right)$.

Let $\mathbb{E}$ and $\mathbb{F}$ be the bundles associated to $P_{M}$ and $\mathbb{R}^{n} \times G$, respectively. The typical fibres are respectively $E$ and $F$. Clearly $\mathbb{F}=\mathbb{R}^{n} \times F$, while as $\mathbb{E}$ needs not to be trivial. Recall from sec. 1 that $\operatorname{Ind}(\mathbb{E}, \mathbb{F})$ is the collection of all embeddings of $\mathbb{E}$ into $\mathbb{F}$ induced by the principal embeddings in $E\left(P_{M}, \mathbb{R}^{n} \times G\right)$. For its relation to $E\left(M, \mathbb{R}^{n}\right)$ we refer to sec.1.

By a stress form on $\operatorname{Ind}\left(\mathbb{E}, \mathbb{R}^{n} \times F\right)$ we mean a map

$$
A: \operatorname{Ind}\left(\mathbb{E}, \mathbb{R}^{n} \times F\right) \longrightarrow A^{1}\left(\mathbb{E}, T\left(\mathbb{R}^{n} \times F\right)\right)
$$

where $A(\bar{J}(j))$ covers $\bar{J}(j)$ for each $\bar{J}(j) \in \operatorname{ind}(\mathbb{E}, \mathbb{F})$. Given a stress form $A$ on $\operatorname{Ind}(\mathbb{E}, \mathbb{F})$ then any smooth map $L: \mathbb{E} \longrightarrow T\left(\mathbb{R}^{n} \times F\right)$, covering $\bar{J}(j)$, regarded as an infinitesimal deformation of $\bar{J}(j)$ yields the virtual work

$$
\begin{aligned}
\mathcal{F}(\bar{J}(j))(L) & :=\int_{\mathbb{E}} A(\bar{J}(j)) \bullet \nabla L \mu(\bar{J}(j)) \\
& =\int_{\mathbb{E}}\left(\nabla(\bar{J}(j))^{*} A(\bar{J}(j)), L\right) \mu(\bar{J}(j)) .
\end{aligned}
$$

The force density $\nabla(\bar{J}(j))^{*} A(\bar{J}(j))$ depends on the particular configuration $\bar{J}(j)$ and hence on the microstructures (if $\operatorname{dim} F \neq 0$ ) possibly in a non-local way.

We could evaluate

$$
\int_{\mathbb{E}}\left(\nabla(J(j))^{*} A(\bar{J}(j)), L\right) \mu(\bar{J}(j))
$$

by integrating over the fibres first and then over $M$. This yields a form on $M$. We proceed in this way below in a somewhat other setting (cf. (3.6)).

Solving for any $\bar{J}(j) \in \operatorname{Ind}\left(\mathbb{E}, \mathbb{R}^{n} \times F\right)$ the equation

$$
\Delta(\bar{J}(j))^{*} A(\bar{J}(j))=\Delta(\bar{J}(j)) \mathcal{H}_{F}(\bar{J}(j))
$$

with the smooth solution $\mathcal{H}(\bar{J}(j))$ (covering $\bar{J}(j))$ ) which is $L_{2}$-perpendicular to the kernel of $\Delta(\bar{J}(j))$ immediately yields (cf. [Bi3]): 


\section{Theorem 3.1}

The stress form $A$ of the deformable medium with microstructures determine the same force densities as the map

$$
\mathcal{H}: \operatorname{Ind}\left(\mathbb{E}, \mathbb{R}^{n}, F\right) \longrightarrow C^{\infty}\left(P_{M}, T\left(\mathbb{R}^{n} \times F\right)\right)
$$

does. In fact we have

$$
\mathcal{F}(\bar{J}(j))(L)=\int_{\mathbb{E}} \nabla \mathcal{H}(\bar{J}(j)) \bullet \nabla L \mu(\bar{J}(j))
$$

for any $\bar{J}(j) \in E\left(\mathbb{E}, \mathbb{R}^{n} \times F\right)$ and any $L \in C_{\bar{J}(j)}^{\infty}\left(\mathbb{E}, T\left(\mathbb{R}^{n} \times F\right)\right)$.

The following theorem shows that we may have a more resolved description of our medium if we define the stress form on $P_{M}$, assumed to be oriented, rather than on its associated bundle.

Let $\mathbb{E}$ and $\mathbb{F}$ be the associated fibre bundles to the $H$-resp. $G$-principal bundles $P_{M}, P_{N}$ as in sec.1, $\chi: P_{N} \times F \rightarrow \mathbb{F}$ the defining quotient maps. Furthermore let $y \in F$ a point in $F$ which is the image of $z \in E$ under the inclusion map $i: E \hookrightarrow F$.

\section{Theorem 3.2}

a) Every $T P_{N}$-valued 1-form $\beta \in A^{1}\left(P_{M}, T P_{N}\right)$ on $P_{M}$ induces a 1 -form $\bar{\beta} \in$ $A^{1}(\mathbb{E}, T \mathbb{F})$ defined by

$$
\bar{\beta}(X)(e):=T \chi_{y}(\beta(V)(p)),
$$

where the $\operatorname{map} \chi_{z}: P_{M} \rightarrow \mathbb{E}$ is the quotient map $\chi$ with fixed second argument, $X:=T \chi_{z}(V)$ a vector in $T \mathbb{E}$ and $e \in \mathbb{E}_{q}$ an element of the fibre of $\mathbb{E}$ above $q \in M$.

b) The correspondence $\beta \mapsto \bar{\beta}$ is many-to-one, more precisely all $\beta \in$ $A^{1}\left(P_{M}, T P_{N}\right)$ which can be transferred into the same $\beta_{0} \in A^{1}\left(P_{M}, T \underline{P}_{N}\right)$ by pullback under the right action $R_{g}: P_{M} \rightarrow P_{N}$ induce the same $\bar{\beta} \in$ $A^{1}(\mathbb{E}, T \mathbb{F})$ with $g \in G$.

Let $A_{H}^{1}\left(P_{M}, \mathbb{R}^{n} \times \mathbf{g}\right)$ be the collection of all the $H$-equivariant smooth one-forms on $P_{M}$ with values in $\mathbb{R}^{n} \times \mathbf{g}$. By a stress form on $E\left(P_{M}, \mathbb{R}^{n} \times G\right)$ we mean a map

$$
A_{P}: E\left(P_{M}, \mathbb{R}^{n} \times G\right) \longrightarrow A_{H}^{1}\left(P_{M}, \mathbb{R}^{n} \times \mathbf{g}\right)
$$

for which $A_{P}(J(j))$ is $H$-equivariant for any $J(j) \in E\left(P_{M}, \mathbb{R}^{n} \times G\right)$. We will touch the special structure of the domain of $A_{P}$ as pointed out in theorem 1.5:

Let $\omega_{0}$ be the connection from on $P_{M}$ inducing the Levi-Cività connection $\nabla$ on $\mathbb{R}^{n} \times F$. Using the horizontal distribution of $\omega_{0}$, any principal embedding $J(j) \in E\left(P_{M}, \mathbb{R}^{n} \times G\right)$ defines a horizontal distribution $\mathbf{H} P_{M}$ on $P_{M}$ which in turn defines a connection form $\omega(J(j))$ on $P_{M}$. Let us denote the vertical bundle on $P_{M}$ by $\mathbf{V} P_{M}$. Restricting $A_{P}(J(j))$ to $\mathbf{V} P_{M}$ and $\mathbf{H} P_{M}$ yields respectively the $H$-equivariant forms $A_{P}^{\mathbf{V}}(J(j))$ and $A_{P}^{\mathbf{H}}(J(j))$. We refer to $A_{P}^{\mathbf{V}}$ and $A_{P}^{\mathbf{H}}$ as the vertical resp. horizontal parts of $A_{P}$. This immediately yields 


\section{Lemma 3.3}

Let $p r_{\mathbb{R}^{n}}$ and $p r_{\mathbf{g}}$ denote the canonical projection from $\mathbb{R}^{n} \times \mathbf{g}$ onto $\mathbb{R}^{n}$ and $\mathbf{g}$, respectively, then $A_{\mathbb{R}^{n}}^{\mathbf{H}}:=\operatorname{pr}_{\mathbb{R}^{n}} \circ A_{P}^{\mathrm{H}}$ defines a one-form $\alpha\left(A_{P}^{\mathbf{H}}(J(j))\right): T M \rightarrow$ $\mathbb{R}^{n}$ as follows

$$
\alpha(J(j)) X(\pi(p)):=\left[p, A_{\mathbb{R}^{n}}^{\mathbf{H}}(J(j)) X^{\mathbf{H}}(p)\right]
$$

for all $X \in \Gamma T M$ and any $p \in P_{M}$.

Unless $A_{P}^{\mathbf{H}}$ is $\mathcal{G}_{G}$-invariant, $\alpha(J(j))$ depends on $J(j)$ and not only on $j$. Hence $\alpha(J(j))$ reflects the internal $H$-symmetry. If $A_{P}^{\mathbf{H}}$ is $\mathcal{G}_{G}$-invariant, then $\alpha(J(j))$ depends on $j$ only and thus neglects the microscopic structures.

To formulate the virtual work of $A_{P}$ we assume the existence of a right invariant metric $\langle,\rangle_{r}$ on $G$ and if preferred we may require that the metric $\langle,\rangle_{F}$ on $F$ restricted to any orbit of $G$ is determined by $\langle,\rangle_{r}$. Lifting $\langle$,$\rangle , the metric on \mathbb{R}^{n}$ (with the help of $\omega_{0}$ ) onto $\mathbb{R}^{n} \times G$, yields together with $\langle,\rangle_{r}$ a metric $($,$) on$ all of $\mathbb{R}^{n} \times G$ given as follows: For any $v, w \in T_{(z, g)}\left(\mathbb{R}^{n} \times G\right)$ let

$$
(v, w):=\left\langle T \pi^{\mathbb{R}} v, T \pi^{\mathbb{R}^{n}} w\right\rangle+\left\langle\omega_{0} v, \omega_{0} w\right\rangle_{r} .
$$

Pulling back (, ) by $J(j)$ yields the metric $m(J(j))$ on $P_{M}$. Let $\mu(J(j))$ be the Riemannian volume associated with $m(J(j))$ and the orientation. Given a stress form $A_{P}(J(j))$ on $P_{M}$ then any $H$-equivariant smooth map $L: P_{M} \longrightarrow \mathbb{R}^{n} \times \mathbf{g}$ being an infinitesimal deformation of $J(j)$ yields the virtual work

$$
\begin{aligned}
\mathcal{F}_{P}(J(j))(L) & =\int_{P_{M}} d A_{P}(J(j)) \bullet d L \mu(J(j)) \\
& =\int_{P_{M}}\left(\nabla(J(j))^{*} A_{P}(J(j)), L\right) \mu(J(j))
\end{aligned}
$$

where $\nabla(J(j))$ denotes the Levi-Cività connection on $P_{M}$ determined by $m(J(j))$. The following is an immediate consequence from the definition of the virtual work.

\section{Lemma 3.4}

For each $J(j) \in E\left(P_{M}, \mathbb{R}^{n} \times \mathbf{g}\right)$ and each $l \in C^{\infty}\left(M, \mathbb{R}^{n}\right)$ the virtual work caused by $l \circ \pi$ is

$$
\begin{aligned}
\mathcal{F}_{P}(J(j))\left(l \circ \pi^{M}\right) & =\int_{P_{M}} A_{P}(J(j)) \bullet d(l \circ \pi) \mu(J(j)) \\
& =\int A_{P}^{\mathbf{H}}(J) \bullet d(l \circ \pi) \mu(j) \\
& =\int_{M}\left\langle\int_{P_{M}} \nabla(J(j))^{*} A_{\mathbb{R}^{n}}^{\mathbf{H}}(J(j)), l\right\rangle \mu(j)
\end{aligned}
$$

where $f$ denotes the fibre integral over $P_{M}$.

Let us continue with our general studies. Solving the equations

$$
\Delta(J(j))^{*} A P(J(j))=\Delta(J(j)) \mathcal{H}_{P}(J(j))
$$

and

$$
\Delta(j)^{*} a(J(j))=\Delta(j) \hbar_{P}(J(j))
$$


for any $J(j) \in E\left(P_{M}, M \times G\right)$ with solutions $\mathcal{H}_{P}(J(j))$ and $\eta_{P}(J(j))$ being $L_{2^{-}}$ perpendicular to $\mathbb{R}^{n} \times \mathbf{g}$ resp. $\mathbb{R}^{n}$ immediately yields

\section{Theorem 3.5}

The properties of the deformable medium with internal symmetries characterized by the stress forms $A_{P}$ and $\alpha\left(A^{\mathbf{H}}\right)$ determine the same force densities as the $H$ equivariant maps

$$
\mathcal{H}_{P}: E\left(P_{M}, \mathbb{R}^{n} \times G\right) \longrightarrow C^{\infty}\left(P_{M}, \mathbb{R}^{n} \times \mathbf{g}\right)
$$

and

$$
h_{P}: E\left(P_{M}, \mathbb{R}^{n} \times G\right) \longrightarrow C^{\infty}\left(M, \mathbb{R}^{n}\right)
$$

do.

\section{Influence of the curvature of $P_{M}$ to the virtual work in a special situation}

Let $A$ be a stress form on $\operatorname{Ind}(\mathbb{E}, \mathbb{F})$. Thus for any $\bar{J}(j) \in \operatorname{Ind}(\mathbb{E}, \mathbb{F})$ the map $A(\bar{J}(j)): T \mathbb{E} \rightarrow T\left(\mathbb{R}^{n} \times F\right)$ is a bundle homomorphism covering $\bar{J}(j)$. Let $\overline{p r}_{\mathbb{R}^{n}}: \mathbb{R}^{n} \times F$ be the projection onto the first factor $\mathbb{R}^{n}$. Then

$$
\operatorname{Tpr}_{\mathbb{R}^{n}} \circ A(J(j)): T \mathbb{E} \longrightarrow T \mathbb{R}^{n}=\mathbb{R}^{n} \times \mathbb{R}^{n}
$$

yields a one-form $A_{\mathbb{R}^{n}}(\bar{J}(j))$ with values in $\mathbb{R}^{n}:$ Let $p r_{2}: \mathbb{R}^{n} \times \mathbb{R}^{n} \rightarrow \mathbb{R}^{n}$ be the projection onto the second factor. The one-form mentioned is defined by

$$
A_{\mathbb{R}^{n}}(\bar{J}(j)):=p r_{2} \circ T \overline{p r}_{\mathbb{R}^{n}} \circ A(\bar{J}(j)): T \mathbb{E} \longrightarrow \mathbb{R}^{n} .
$$

Similarly let $\overline{p r}_{F}: \mathbb{R}^{n} \times F \rightarrow F$ be the projection onto the second factor $F$. Then

$$
A_{F}(\bar{J}(j)):=T \overline{p r}_{F} \circ A(\bar{J}(j)) \quad \forall \bar{J}(j) \in \operatorname{Ind}(\mathbb{E}, \mathbb{F}) .
$$

Thus if we write $T\left(\mathbb{R}^{n} \times F\right)=\mathbb{R}^{n} \times \mathbb{R}^{n} \times T F$ we find

$$
A(\bar{J}(j))=\left(j, A_{\mathbb{R}^{n}}(\bar{J}(j)), A_{F}(\bar{J}(j))\right) .
$$

In this section we show that given any stress form $A$ on $\operatorname{Ind}(\mathbb{E}, \mathbb{F})$ an influence of the curvature $\Omega(\bar{J}(j))$ of $\omega(\bar{J}(j))$ is detectable in the form $A_{\mathbb{R}^{n}}$ and hence in its exact part $d \mathcal{H}_{\mathbb{R}^{n}}(\bar{J}(j)): T M \longrightarrow \mathbb{R}^{n}$ for any $\bar{J}(j) \in E\left(P_{M}, \mathbb{R}^{n} \times F\right)$. Here $\omega(\bar{J}(j))$ is the connection determined in such a way that its horizontal distribution on $\mathbb{F}$ is mapped to the one determined by $\omega_{0}$. Recall that $\omega_{0}$ is the connection form yielding the Levi-Cività connection on $F$.

To this end we use $G \subset S O(n)$ and $H \subset S O(\operatorname{dim} M)$. The curvature $\Omega(\bar{J}(j))$ of $\omega(\bar{J}(j))$ is defined by

$$
\Omega(\bar{J}(j)):=d_{\omega(\bar{J}(j))} \omega(\bar{J}(j)),
$$

where $d_{\omega(\bar{J}(j))}$ is the covariant exterior differential determined by $\omega(\bar{J}(j))$.

We will study the influence of $\Omega(\bar{J}(j))$ as far as it is reflected in a bundle map $\rho_{\Omega}(\bar{J}(j))$ of $T M$ caused by $R^{\perp}(\bar{J}(j))$, both to be defined below. The map $\Omega(\bar{J}(j))$ may naturally be identified with a h-valued two-form on $M . R_{\Omega}^{\perp}$ defined by

$$
R_{\Omega}^{\perp}(\bar{J}(j))\left(u_{q}, v_{q}\right) w_{q}:=\Omega(\bar{J}(j))\left(u_{q}, v_{q}\right)\left(w_{q}\right)
$$


for all $u_{q}, v_{q}, w_{q} \in \Gamma(T M)$ and for all $q \in M$ where the skew transformation $\Omega(\bar{J}(j))$ in (4.1) is supposed to operate for any $q \in M$ on $T_{q} M$ as the elements of $\operatorname{so}(\operatorname{dim} M)$ do. Thus it operates analogously as $\Omega(j)_{\text {Riem }}$ does, the two-form determining the curvature of the Riemannian metric on $M$. The form $R^{\perp}(\bar{J}(j))$ yields the $\mathbb{R}$-valued two-tensor (cf. example (ii) in sec.5).

$$
\rho_{\Omega}(\bar{J}(j))(Y, Z):=\operatorname{tr}_{X} R_{\Omega}^{\perp}(\bar{J}(j))(X, Y) Z \quad \forall Y, Z \in \Gamma(T M) .
$$

Therefore we find a bundle map $r_{\Omega}(\bar{J}(j)): T M \rightarrow T M$ over id such that

$$
m(j)\left(r_{\Omega}(\bar{J}(j)) X, Y\right)=\rho_{\Omega}(\bar{J}(j))(X, Y) \quad X, Y \in \Gamma(T M) .
$$

Following the method presented in the equation (2.2) $d j \bar{r}_{\Omega}(\bar{J}(j))$ determines in turn a smooth $h_{\Omega}(\bar{J}(j)): M \rightarrow \mathbb{R}^{n}$, the exact part of $d j \bar{r}_{\Omega}(\bar{J}(j))$.

Taking the $L_{2}$-component with respect to the dot metric of $A_{\mathbb{R}^{n}}(\bar{J}(j))$ along $d j \bar{\rho}_{\Omega}(\bar{J}(j)) \circ \pi^{\mathbb{E}}=\left(\pi^{\mathbb{E}}\right)^{*} d j \bar{\rho}_{\Omega}(\bar{J}(j))$ with $\pi^{\mathbb{E}}: \mathbb{E} \rightarrow M$ the projection, yields a real number $s(\bar{J}(j))$ such that

$$
A_{\mathbb{R}^{n}}(\bar{J}(j))=s(\bar{J}(j)) \cdot d j \bar{\rho}_{\Omega}(\bar{J}(j)) \circ T \pi^{\mathbb{E}}+\text { rest }
$$

for all $J(j) \in \operatorname{Ind}\left(\mathbb{E}, \mathbb{R}^{n} \times F\right)$. Since the virtual work caused by $A$ splits additively into the virtual work, caused by $A_{\mathbb{R}^{n}}$ and $A_{F}$, we thus have:

\section{Theorem 4.1}

The curvature of the connection as far as it is reflected in $\bar{\rho}_{\Omega}(\bar{J}(j))$ influences $A(\bar{J}(j))$ and the virtual work $F$ through the real $s(\bar{J}(j))$ for each $\bar{J}(j) \in$ $E\left(P_{M}, P_{\mathbb{R}^{n}}\right)$.

This shows that distorting the geometric setting of microstructures may cause virtual work (cf. sec.5 (ii)).

\section{Remark}

If $H=S O(\operatorname{dim} M)$ and $\mathbb{E}$ the unit sphere bundle of $T M$ then $\rho_{\Omega}(\bar{J}(j))$ is the Ricci tensor $\operatorname{Ric}(j)$ of the metric $m(j)$ on $M$ (cf. [Bi2]).

\section{Simple Examples}

(i) In $[\mathrm{MH}]$ we find the description of configurations of Cosserat media in $\mathbb{R}^{3}$ : The manifold $F$ is either $\mathbb{R}^{3}$, the unit sphere $S^{2}$ or the real projective space $\mathbb{R} P^{3}$. We set $E:=\{e\}$, that is a zero dimensional submanifold of $F$. As a configuration we consider a bundle map

$$
M \times\{e\} \stackrel{J(j)}{\longrightarrow} \mathbb{R}^{3} \times F
$$

being an embedding over an embedding $j \in E\left(M, \mathbb{R}^{3}\right)$. Thus the diagram

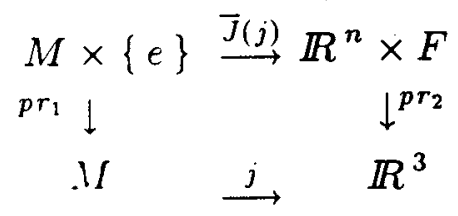


commutes. Our sorts of configurations in this context are configurations in the sense of $[\mathrm{MH}]$ as easily seen.

(ii) The next kind of (a rather theoretical) example is inspired by the description of lipid in water studied in the book [deG]. We idealize the situation in the following way: Let $\operatorname{dim} M=2$. At each point $q \in M$ there is a disc attached. We make this picture more precise: Let $\mathbb{R}^{2} \subset \mathbb{R}^{n}$. We consider $F:=\{x \in$ $\left.\mathbb{R}^{n} \mid\|x\|=1\right\} \equiv S^{n-1}$ and $E:=\left\{x \in \mathbb{R}^{2} \mid\|x\| \equiv 1\right\}=S^{1}$. Thus $E \subset F$ is closed and $F$ is compact. The group $G$ is $S O(n)$ and $H$ is chosen to be $S O(2)$. If we let $P_{M}$ be the frame bundle of $T M$, an $S O(2)$-principal bundle, then $\mathbb{E}$ is a subbundle of $T M$, in fact it is the unit sphere bundle of $T M$. If we let, however, $P_{M}$ be the orthogonal frame bundle of a plane bundle $\mathbb{L}$ (a complex line bundle) equipped with a bundle metric then clearly this bundle may differ from $T M$. The bundle $\mathbb{E}$ is the unit sphere bundle of $\mathbb{L}$. Given $\bar{J}(j) \in \operatorname{ind}\left(\mathbb{E}, \mathbb{R}^{n} \times S^{n-1}\right)$ over $j \in E\left(M, \mathbb{R}^{n}\right)$ we pull back the product metric $\langle$,$\rangle on \mathbb{R}^{n} \times S^{n-1}$ (each factor carries the metric given by the fixed scalar product on $\left.\mathbb{R}^{n}\right)$. This yields $m(\bar{J}(j))$.

The free-energy (cf. [deG]) in our situation certainly depends on a configuration $\bar{J}(j) \in \operatorname{ind}\left(\mathbb{E}, \mathbb{R}^{n} \times S^{n-1}\right)$ of the line bundle $\mathbb{L}$. We extend $\bar{J}(j)$ in the obvious way to a bundle embedding of $\mathbb{L}$ into $\mathbb{R}^{n} \times \mathbb{R}^{n}$. This extension is denoted by $\bar{J}(j)$, too. The metric on $\mathbb{L}$ determined by the embedding $\bar{J}(j)$ of $\mathbb{L}$ into $\mathbb{R}^{n} \times \mathbb{R}^{n}$ is also denoted $m(\bar{J}(j))$.

Our first goal is to describe the various types of the bundle $\mathbb{L}$. To this end we need to study the curvature given by the metric $m(J(j))$, a characteristic ingredient of $\mathbb{L}$ (cf. [K] or $[\mathrm{Sn}]$ ). The Levi-Cività connection $\nabla(\bar{J}(j))$ on $\mathbb{L}$ of $m(\bar{J}(j))$ is determined by a connection form $\omega(\bar{J}(j))$ on $P_{M}$ of which its curvature $\Omega(\bar{J}(j))$ is a horizontal and $S O(2)$-equivariant two-form on $P_{M}$ with values in so(2). $\Omega(\bar{J}(j))$ defines a cohomology class in $H^{2}(M, \mathbb{R}) \cong \mathbb{R}$ which can be identified with an integral cohomology class and vice versa each integral class defines a complex line bundle by a theorem of Weil (cf.[Sn] or $[\mathrm{Ko}]$ ). (It is the same kind of class Professor Doebner uses in his description of quantization.) This class has to be the same for all $\bar{J}(j)$ in a connected component of ind $(\mathbb{E}, \mathbb{F})$.

Before we are going to specify a stress form (cf. (3.1) to (3.3) we continue with the surroundings of the real $s(\bar{J}(j))$ in (4.1). The curvature $R_{\Omega}^{\perp}$ of $\nabla(\bar{J}(j))$ as introduced in (4.1) is given by

$$
R_{\Omega}^{\perp}(X, Y) Z=\Omega(\bar{J}(j))\left(X^{h o r}, Y^{h o r}\right)(Z)
$$

where $X, Y, Z \in \Gamma T M$ and where the upper index hor means the horizontal lift. Clearly $R_{\Omega}^{\frac{1}{\Omega}}$ is not the curvature of the metric $m(j)$.

Now let us perform $\operatorname{djr}_{\Omega}(\bar{J}(j)): T M \rightarrow \mathbb{R}^{n}$ as done in sec.4. To understand the curvature $\Omega(\bar{J}(j))$ in view of $(4.4)$ we regard it as a $\mathbb{R}$-valued two-form (via the canonical isomorphisms of so(2) to $\mathbb{R}$ ) and call it $\Omega^{\perp}(\bar{J}(j))$ if it operates in the sense of $(4.1)$ and (5.1). Clearly $\Omega^{\perp}(\bar{J}(j))=\Omega(\bar{J}(j))$ in case of $\mathbb{L}=T M$. Next we write

$$
\Omega^{\perp}(\bar{J}(j))=\psi(\bar{J}(j)) \cdot \mu(j)
$$

with $\psi(\bar{J}(j)) \in C^{\infty}(M, \mathbb{R})$. Since (due to $\operatorname{dim} M=2$ )

$$
\Omega_{R i \in m}(j)=\frac{\lambda(j)}{4 \pi} \cdot \mu(j)
$$


(cf. [D]) with $\lambda(j)$ the trace of the Ricci tensor $\operatorname{Ric}(j)$ of $m(j)$, we deduce

$$
\frac{\lambda(j)}{4 \pi} \cdot \Omega(\bar{J}(j))=\frac{\lambda(j)}{4 \pi} \cdot \psi(\bar{J}(j)) \cdot \mu(j) .
$$

Since

$$
\psi(\bar{J}(j)) \cdot \Omega_{R i e m}(j)=\frac{\lambda(j)}{4 \pi} \cdot \Omega^{\perp}(\bar{J}(j))
$$

we find due to $\operatorname{dim} M=2$ again (cf. (4.2))

$$
\psi(\bar{J}(j)) \cdot \operatorname{Ric}(j)=\frac{1}{2} \cdot \psi \cdot(\bar{J}(j)) \lambda(j) \cdot m(j)=\frac{\lambda(j)}{4 \pi} \cdot \rho_{\Omega}(\bar{J}(j)) .
$$

Taking traces with respect to the metric $m(j)$ on both sides and taking in account that the scalar curvature of $\mathrm{m}(\mathrm{j})$ varies smoothly with $j \in E\left(M, \mathbb{R}^{n}\right)$ yields (cf. (4.3))

$$
\psi(\bar{J}(j))=\frac{1}{4 \pi} \cdot \operatorname{tr} r_{\Omega}(\bar{J}(j)) \quad \text { and } \quad r_{\Omega}(\bar{J}(j))=\frac{1}{2} \cdot\left(\operatorname{tr} r_{\Omega}(\bar{J}(j))\right) \cdot \mathrm{id}
$$

(which is obvious if $\lambda(j)$ is a nowhere vanishing function). This shows

$$
\Omega^{\perp}(\bar{J}(j))=\frac{1}{4 \pi} \cdot \operatorname{tr} r_{\Omega}(\bar{J}(j)) \cdot \mu(j)
$$

Let $[\beta]$ denote the cohomology class of the closed form $\beta$. Since $a \cdot[\Omega]=k \cdot 1 \in$ $\mathbb{Z}$ for some $a \in \mathbb{R}$ we conclude

$$
a \cdot\left[\Omega^{\perp}(\bar{J}(j))\right]=\frac{a}{4 \pi} \cdot\left[\operatorname{tr} \rho_{\Omega}(\bar{J}(j)) \cdot \mu(j)\right]=k \cdot b \cdot\left[\frac{\mu(j)}{\mathcal{V}(j)}\right]
$$

with $b \in \mathbb{R}$ and $\mathcal{V}(j):=\int \mu(j)$ for any $j \in E\left(M, \mathbb{R}^{n}\right)$. In turn (5.4) yields

$$
\int_{M} \operatorname{tr} \rho_{\Omega}(\bar{J}(j)) \mu(j)=4 \pi \cdot k \cdot \frac{b}{a} \quad a \neq 0 .
$$

(5.5) shows that $\operatorname{tr} \rho_{\Omega}(\bar{J}(j))$ does not vanish in general.

Hence the exact part $\eta_{\Omega}(\bar{J}(j))$ in $(4.4)$ is the exact part of $\frac{1}{2} \operatorname{tr} \rho_{\Omega}(j) \cdot d j$ in this example. Even though $r_{\Omega}(\bar{J}(j))$ is not trivial the real number $s(\bar{J}(j))$, depending on the stress form, still may vanish.

Next we will produce a kind of stress form by starting from the deformation free-energy density (cf. [LL], [StSt] and [deG]). The deformation free-energy density depends on a director field, that is on a vertical field $\mathbf{n}$ on $\mathbb{L}$. This field restricted to the unit sphere in the fibre $\mathbb{L}_{q}$ of $\mathbb{L}$ with $q \in M$ maps into $\mathbb{L}_{q}$. Now we suppose that $\mathbf{n}(\bar{J}(j)):=d \bar{J}(j) \mathbf{n}$ is a unit normal field along $\bar{J}(j)$. Hence $m(\bar{J}(j))(\mathbf{n}, \mathbf{n})=1$. The $\mathbb{R}^{n} \times \mathbb{R}^{n}$-valued one-form yields a Weingarten map $W(\bar{J}(j))$, a strong bundle endomorphism of $T \mathbb{L}$ satisfying

$$
d J(j) W(\bar{J}(j)) \xi=\nabla_{\xi}(\bar{J}(j)) \mathbf{n} \quad \forall \xi \in \Gamma T \mathbb{L} .
$$

We assume in our theoretical example that the (smooth) deformation freeenergy density $\mathbf{f}_{d}$ depends at $\bar{J}(j)$ on $W(\bar{J}(j))$ only. We expand $\mathbf{f}_{d}$ at $\bar{J}(j)$ up to second order and follow the argument in [deG] to avoid linear terms. For simplicity we let $\mathbf{f}_{d}$ be of the form

$$
\mathbf{f}_{d}(\bar{J}(j))=K \cdot \operatorname{tr} W(\bar{J}(j))^{2}
$$


with $K$ a positive constant (a special case of the deformation free-energy density defined in $[\mathrm{StSt}]$ or $[\mathrm{deG}])$ and the deformation free-energy $\mathbf{f}_{t o t}$ hence is

$$
\begin{aligned}
\mathbf{f}_{\text {tot }}(\bar{J}(j)) & =K \cdot \int_{\mathbb{E}} \operatorname{tr} W(\bar{J}(j))^{2} \mu(\bar{J}(j)) \\
& =K \cdot \int_{\mathbb{E}} \nabla(\bar{J}(j)) \mathbf{n} \bullet \nabla(\bar{J}(j)) \mathbf{n} \mu(\bar{J}(j)) .
\end{aligned}
$$

Here $\mathcal{V}_{\mathbb{E}}(\bar{J}(j))$ denotes the Riemannian volume of $\mathbb{E}$ with respect to $m(\bar{J}(j))$. Differentiating in $L\left(\mathbb{L}, \mathbb{R}^{n} \times \mathbb{R}^{n}\right)$ the map $\mathbf{f}_{\text {tot }}$ at $\bar{J}(j)$ with respect to $L$ we find

$$
\begin{aligned}
\mathrm{d} \mathbf{f}_{\text {tot }}(\bar{J}(j))(L)=-2 \cdot K \cdot \int_{\mathbb{E}} d \bar{J}(j) W(\bar{J}(j))^{2} \bullet d L \mu(\bar{J}(j)) \\
\quad+2 \cdot K \cdot \int_{\mathbb{E}} \nabla(\bar{J}(j)) \mathbf{n} \bullet \nabla(\bar{J}(j)) \mathbf{n} \cdot \frac{1}{2} \operatorname{tr}_{m(\bar{J}(j))} \mathrm{d} m(\bar{J}(j))(L) \mu(\bar{J}(j)) \\
=K \cdot \int_{\mathbb{E}}\left(-2 \cdot d \bar{J}(j) W(\bar{J}(j))^{2}+2 \cdot\left(\operatorname{tr} W(\bar{J}(j))^{2}\right) \cdot d \bar{J}(j)\right) \bullet d L \mu(\bar{J}(j)) .
\end{aligned}
$$

Setting

$$
A(\bar{J}(j)):=2 \cdot K \cdot d \bar{J}(j)\left(\operatorname{tr} W\left(\bar{J}(j)^{2}\right) \cdot \mathrm{id}-W(\bar{J}(j))^{2}\right)
$$

yields

$$
\mathrm{d} \mathbf{f}_{t o t}(\bar{J}(j))(L)=\int_{\mathbb{E}} A(\bar{J}(j)) \bullet d L \mu(\bar{J}(j))=\int_{\mathbb{E}}\left\langle\nabla(\bar{J}(j))^{*} \alpha(\bar{J}(j)), L\right\rangle \mu(\bar{J}(j))
$$

for each $J(j) \in \operatorname{ind}\left(\mathbb{E}, \mathbb{R}^{n} \times S^{n-1}\right)$. The exact part of $\alpha(\bar{J}(j))$, a vector field along $\bar{J}(j)$ ) with values in $\mathbb{R}^{n} \times \mathbb{R}^{n}$ characterizes the medium at hand.

Clearly $\mathrm{d} \mathbf{f} \mathbf{f}_{t o t}$ is not the virtual work in general. However if $L$ is an isothermal distortion $\mathrm{d} \mathbf{f}_{\text {tot }}(\bar{J}(j))(L)$ is a virtual work (cf. [LL]).

Finally the real $s(\bar{J}(j))$ in theorem 4.1 is due to (4.4), (5.2) and (5.4) given by

$$
\begin{aligned}
s(\bar{J}(j)) \cdot & \int_{\mathbb{E}}\left(\operatorname{tr} r_{\Omega}(\bar{J}(j))\right)^{2} \mu(\bar{J}(j)) \\
= & 4 \cdot \int_{\mathbb{E}} \operatorname{tr} W(\bar{J}(j))^{2} \cdot \operatorname{tr} r_{\Omega}(\bar{J}(j)) \mu(\bar{J}(j)) \\
& -2 \cdot \int_{\mathbb{E}} \operatorname{tr}\left(W(\bar{J}(j))^{2}\right)^{h o r} \cdot \operatorname{tr} r_{\Omega}(\bar{J}(j)) \mu(\bar{J}(j))
\end{aligned}
$$

for any $\bar{J}(j)) \in \operatorname{ind}(\mathbb{E}, \mathbb{F})$. The restriction of $W(\bar{J}(j))^{2}$ to the horizontal distribution is denoted by $\left(W(\bar{J}(j))^{2}\right)^{h o r}$. 


\section{Appendix: The dot-product}

Here we want to explain the dot-product, cf. sec.2. Using the notation of sec.1 let $M$ be a smooth compact oriented manifold of finite dimension and $j \in E(M, N)$ a given embedding of $M$ into $N$, where $\operatorname{dim} M \leq \operatorname{dim} N$.

We split an $T N$-valued 1 -form $\gamma \in A^{1}(M, T N)$ covering $j$ in the following way:

$$
\gamma(X)=(c(\gamma, T j) \circ T j+T j \circ A(\gamma, T j))(X), \quad X \in \Gamma(T M),
$$

where $A(\gamma, T j)$ is a strong bundle morphism of $T M$ and $c(\gamma, T j)$ a bundle morphism of $T N^{n} \mid j(M)$, which is skew adjoint with respect to to the euclidean metric and which maps $T j T M$ into its normal bundle and vice versa. So $c(\gamma, T j)$ is a infinitesimal version of the well known Gauss-map (cf. eq. [Bi3]). Let $A_{E}(M, T N)$ consists of all $\gamma \in A^{1}(M, T N)$ covering embeddings in $E(M, N)$. Clearly $A_{E}\left(M, T \mathbb{R}^{n}\right)=E\left(M, \mathbb{R}^{n}\right) \times A^{\mathbf{1}}\left(M, \mathbb{R}^{n}\right)$ in case of $N=\mathbb{R}^{n}$.

\section{Definition}

For any $j \in E(M, N)$ and any $\alpha, \beta \in A^{1}(M, T N)$ covering $j$ the dot product - : $A^{1}(M, T N) \times A^{1}(M, T N) \rightarrow C^{\infty}(M)$ is defined by

$$
\alpha \bullet \beta:=-\frac{1}{2} \operatorname{tr}(c(\alpha, T j) \circ c(\beta, T j))+\operatorname{tr}\left(A(\alpha, T j) \circ A^{*}(\beta, T j)\right) .
$$

Here $A^{*}$ denotes the adjoint of $A$ with respect to $m(j)$.

Consequently this product provides us with an inner product on $A_{E}^{1}(M, T N)$ defined by

$$
\mathscr{g}(j)(\alpha, \beta):=\int_{M} \alpha \bullet \beta \mu(j)
$$

which has the following property (cf. [Bi1] or [Bi3]):

$$
g(j)(\alpha, \nabla l)=\int_{M} \alpha \bullet \nabla l \mu(j)=\int_{M}\left\langle\nabla^{*} \alpha, l\right\rangle \mu(j)
$$

where $\nabla$ denotes the covariant derivative with respect to $m(j)$.

The dot metric $\mathscr{g}(j)$ is hence equivalently described by the usual inner product on $T N$-valued forms defined by

$$
g(j):=\int_{M} \gamma \wedge * \eta
$$

$\wedge$ denotes the wedge multiplication on $T N$-valued forms and $*$ is essentially the Hodge star relative to the Riemannian metric $m(j)$. 


\section{References:}

[A] T. Ackermann: Dissertation Universität Mannheim, to appear

[Bi1] E. Binz: Symmetry, Constitutive Laws of Bounded Smoothly Deformable Media and Neumann Problems; Symmetries in Science V, Ed. B. Gruber, L.C. Biedenharn and H.D. Doebner, Plenum Press, New York (1991)

[Bi2] E. Binz: On the Irredundant Part of the First Piola Kirchhoff Stress Tensor; Rep. of Math. Physics, to appear

[Bi3] E. Binz: Global differential geometric methods in elasticity and hydrodynamics; in: Differential Geometry, Group Representations an Quantisations, LN in Physics 379, Springer-Verlag, Heidelberg (1991)

[BSF] E. Binz, J. Sniatycki, H.-R. Fischer: Geometry of Classical Fields; Math. Studies 154, North-Holland, Amsterdam (1988)

[D] J. Diendonné: Elément d'analyse; tome IX, cahier scientific fascicule, XLII, Gauthier-Villars (1982)

[deG] P.G. de Genne: The Physics of Liquid Crystals; The international series of monographs in physics, Oxford University Press, Oxford, London (1977)

[GHV] W. Greub, S. Halperin, J. Vanstone: Connections, Curvature and Cohomology, I and.II; Acad. press, New York, (1972-73)

[KN] S. Kobayashi, K. Nomizu: Foundations of Differential Geometry Vol. 1\&2; Wiley, New York (1969)

[Ko] B. Konstant: Quantization and Unitary Representation; Lecture Notes in Mathematics 170, Springer Berlin Heidelberg, New York (1970)

[LL] L.D. Landau, E.M. Lifschitz: Lehrbuch der theoretischen Physik VII, Elastizitätstheorie; 6. Auflage, Akademie-Verlag, Berlin (1989)

[MH] J.E. Marsden, J.R. Hughes: Mathematical Foundations of Elasticity; Prentice Hall, Inc. Englewood Clifts, New Jersey (1983)

[P] R.S. Palais: Foundations of Global Non-linear Analysis; Benjamin, New York (1968)

[Sn] J. Sniatycki: Geometric Quantization and Quantum Mechanics; Applied Math. Sciences 30, Springer-Verlag, New York, Heidelberg, Berlin (1980)

[StSt] M.J. Stephen, J.P. Straley: Physics of Liquid Crystals; Review of Modern Physics, Vol.46, No.4 (Oct. 1974) 